\title{
The Subversive Potential of Wikipedia: A Resource for Diversifying Political Science Content Online
}

Claire Timperley, Victoria University of Wellington

ABSTRACT Wikipedia is a powerful tool that reinforces dominant knowledge paradigms, especially those emanating from the Global North. Simultaneously, it has subversive potential to challenge these paradigms, offering a far-reaching platform to voices that have been traditionally marginalized and underserved. Despite this potential, instructors often employ an "abstinence approach" to Wikipedia, telling students to avoid using it in their academic work. This article advocates for active engagement with Wikipedia with the aim of challenging bias resulting from omission or underrepresentation of particular material to develop more diverse, publicly accessible political science content. First, I explain the theoretical objectives of the assignment and describe its structure. Then I reflect on its successes, challenges, and possible modifications.

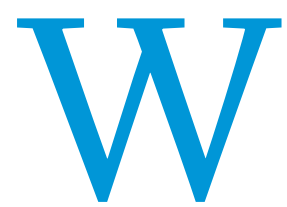
ikipedia is a powerful tool used widely by students and academics as an apparently neutral source of information about many topics. The platform is often lauded for its role in democratizing knowledge through its open-editing approach. Because any individual has the potential to contribute to Wikipedia entries, it serves-at least nominallyto uphold equality among contributors. Moreover, studies have shown that the communal peer-review culture of Wikipedia results in fewer errors than traditional encyclopedias such as the Encyclopedia Britannica (Fallis 2008) and that most contributors offer highly reliable contributions (Anthony, Smith, and Williamson 2009). As one of the most visited websites in the world, Wikipedia also makes its content available to a wide audience. Presentation of its content as factual and neutral confers legitimacy on Wikipedia's content, with search engines perpetuating this perception by presenting Wikipedia content in "fact boxes" at the top of search pages (Ford and Wajcman 2017). Promoted as a platform to share "knowledge," Wikipedia powerfully shapes citizens' understandings of the world.

Despite the relative reliability of the content presented on Wikipedia pages, concern remains about how omissions in the platform's coverage validate topics deemed important by its contributors, skewing context toward coverage of particular subject and geographical areas (e.g., popular culture and the Global North) (Ford and Wajcman 2017). Meanwhile, content related to, for

Claire Timperley ${ }^{(D)}$ is a lecturer in political science at Victoria University of Wellington. She can be reached at claire.timperley@vuw.ac.nz. example, the Global South (Gallert and van der Velden 2015) and women (Gruwell 2015) is underrepresented. This article describes an assignment designed to encourage students to interrogate the content and sources privileged by Wikipedia. The assignment requires students to examine Wikipedia pages related to New Zealand politics and to augment them using their own research. Inviting students to critically examine Wikipedia pages related to content that they explore in their research papers exposes gaps in Wikipedia's coverage. Students are then asked to do something about what they observe by editing entries. Student feedback and my own observations of student development and engagement through this assignment confirm its success. The theoretical underpinnings of this assessment and its key learning objectives are explained before describing the assignment structure and sharing student feedback, challenges, and possible modifications.

\section{THEORETICAL OBJECTIVES}

Globally, there have been calls in academia to "decolonize the syllabus" by redefining what counts as the canon for any particular discipline. ${ }^{1}$ Political science is no exception; however, much work remains to be done across subfields, particularly with respect to indigenous politics and peoples (Bruyneel 2014; Ferguson 2016). In New Zealand, discussion and analysis of colonization are central features of academic discourse, but significant work remains to address structural inequalities within the university and society at large.

A Wikipedia-based assessment emerged from my desire to diversify the New Zealand politics curriculum offered by my department in a way that made students feel invested in this 
objective, as well as to foster robust academic skills and support original research. To motivate and meet the needs of a new generation of students, I was also keen to utilize modern technologies. Inspired by feminist edit-a-thons (aimed at reducing Wikipedia's gender gap), I created an assignment that required students to engage comprehensively with Wikipedia, from reviewing and critiquing its content to contributing their own material. The focus was on generating New Zealand politics content, with particular emphasis on improving availability of material previously underdeveloped on the platform.

In designing this assignment, I drew on a growing body of work that embraces Wikipedia as an important resource in highereducation learning and teaching. Some academics argue that rather than outright rejecting the use of Wikipedia or ignoring its research to an academic audience (in a research paper) and a public audience (in their contribution to Wikipedia).

The assignment consists of the following four parts:

(1) a literature review

(2) a short essay identifying and analyzing edits made to Wikipedia articles

(3) a self-reflection essay

(4) class attendance

In the first weeks of the course, students are assigned to groups of three or four based on similar research topics. These groups enable students to help one another procedurally by troubleshooting technical issues and substantively by receiving peer

\section{...this assignment asks students to see themselves as transitioning from "consumers" to "producers" of knowledge, with much of value to offer a nonspecialist public audience.}

existence-when studies show that most students and academics rely on it for at least basic information gathering (Knight and Pryke 2012) - more can be gained from scrutinizing and contributing to it (Bayliss 2013; Di Lauro and Johinke 2017; Jennings 2008). In political science, Cassell (2018) argued that Wikipedia is a good resource for teaching controversial issues. Kennedy and his co-authors (2015) advocated using Wikipedia in introductory comparative politics courses to develop essential skills, improve student learning and retention, and improve the quality of publicly accessible information. Brown (2011) analyzed the value of Wikipedia as a source of data for political scientists.

Another literature emerging from academic engagement with Wikipedia highlights the consequences of the contributor gender gap, particularly with respect to which content is covered on the platform (Ford and Wajcman 2017; Gruwell 2015; Wagner et al. 2016). Despite the plethora of work considering gender bias, however, there is surprisingly little academic exploration of whether Wikipedia's contributors are representative of other nondominant voices or the ways in which Wikipedia reinforces or challenges dominant knowledge paradigms, especially those emanating from the Global South (exceptions include Gallert and van der Velden 2015 and Wadhwa and Fung 2014).

This article brings these two literatures-using Wikipedia in assessment and problems of representation on the platformtogether to explore different objectives for using Wikipedia as a learning and teaching tool. Rather than focusing on gender, I suggest engaging with Wikipedia to encourage students to think more broadly about privileged epistemologies, as well as providing an opportunity to challenge them through their own contributions to the site. In so doing, I draw attention to the ways that Wikipedia perpetuates certain dominant knowledge paradigms, as well as its distinctive potential to challenge them.

\section{THE ASSIGNMENT}

This assignment is used in an upper-level undergraduate course on New Zealand politics, with enrollment of approximately 80 to 100 students. It is designed to complement students' original research papers: students are expected to hone their expertise in one aspect of New Zealand politics and then present their feedback on their draft and sharing resources related to their topic. Establishing the collaborative nature of the assignment is essential for its success, especially given low levels of student experience with editing and the value of support in navigating Wikipedia's peer-review culture, especially around contentious topics. A significant amount of class time is dedicated to working on this assignment-12 one-hour sessions-which reduces group scheduling problems and ensures that the instructor is available to work through issues individually or collectively.

My own experience in editing Wikipedia was limited when I designed this assignment. Lacking expertise emerged as an advantage. Presenting myself to students as a nonexpert established trust and a willingness for students to share their vulnerabilities and concerns with me, as I did with them. As such, I recommend this assignment to those with little or no experience in editing Wikipedia. Given Wikipedia's ethos that "anyone can edit" the platform, numerous helpful tools are available for instructors and students navigating the process for the first time. These include Wikipedia's own resources, which guide instructors and students through the process of editing, including best-practice suggestions for using the "sandbox" to draft contributions, how to identify and correctly reference sources, and the use of discussion pages to explain and justify edits. ${ }^{2}$

\section{LEARNING OBJECTIVES}

My objectives for the assignment are ambitious and wide ranging. They are described in the following sections.

\section{Interrogate Epistemologies}

Although primarily concerned with students' critical engagement with Wikipedia, this assignment encourages them to interrogate knowledge and sources more broadly across society and to consider whose voices are privileged in which settings.

\section{Public Good}

In her landmark book, Decolonizing Methodologies, Smith (1999) identified the need for research to contribute to decolonization of both academia and society. This assignment takes that 
obligation seriously, bringing to light research cloistered in the "ivory tower" or archives, especially that which attends to marginalized perspectives and knowledge.

\section{Student Engagement}

This is a counterintuitive assignment for many students because they often are told explicitly by instructors not to use Wikipedia. Asking students to think about why and encouraging them to critically engage with (and even embrace) it appeals to students-it is a chance to sample forbidden fruit! Moreover, this assignment asks students to see themselves as transitioning from "consumers" to "producers" of knowledge, with much of value to offer a nonspecialist public audience. For most students, affirmation of their growing expertise is inherently exciting and worthwhile.

\section{Develop Deep Knowledge of Subject Matter}

Complementing the Wikipedia assignment is a requirement to write an original research paper on a topic of a student's choosing. familiarizes students with the structure of Wikipedia pages and encourages them to consider how the presentation and categorization of information may affect what a reader considers to be important. Students are encouraged to closely examine which sources are used to support the claims being made. Once students have selected a topic, they refine their search to identify how Wikipedia content reflects-or fails to reflect-the material and sources they use in their research paper. Much of this work is undertaken collaboratively in groups, but all students write their own literature review. Drafts are peer reviewed before final submission.

\section{Editing and Analyzing Articles}

After identifying gaps in the New Zealand politics content on Wikipedia, students are expected to draw on their original research for their paper to contribute to Wikipedia entries. This task is assessed on their description of what they edited (supported with before and after screenshots 3 ) as well as justification for why they chose particular edits and an explanation of challenges they faced. The task is not assessed primarily

\section{Peer review is the foundational premise of Wikipedia; as a result, students engage-some for the first time-in drafting and redrafting work, as well as having to justify language and content choices to others.}

Focusing on one topic across the course assessment and presenting it in different formats encourages comprehensive understanding of one aspect of New Zealand politics.

\section{Writing Skills}

Writing for Wikipedia requires students to be precise in the language they use, learn how to make verifiable statements, and reference correctly. Peer review is the foundational premise of Wikipedia; as a result, students engage-some for the first time-in drafting and redrafting work, as well as having to justify language and content choices to others.

\section{Understand and Interrogate Sources}

Scrutinizing Wikipedia pages motivates students to interrogate what constitutes a "strong" source, to think about omitted perspectives, and to challenge material rather than take it at face value.

\section{ASSIGNMENT STRUCTURE}

The assignment consists of four parts, each developing key skills to reflect on and effectively edit Wikipedia's content.

\section{Literature Review}

The key objectives of the literature review are to familiarize students with the structure and style of Wikipedia articles, to identify gaps in the content related to New Zealand politics, and to critically reflect on the epistemological commitments present on those pages. To help students start this process, in our first workshop we brainstorm high-quality online sources for New Zealand politics and compare the information presented on these sites (e.g., government websites such as Statistics New Zealand) with related information available on Wikipedia. This activity on the quantity of contributions-students are not required to create entirely new entries (although many did)-but it is expected that a number of clear changes will be made (e.g., adding lines of text and providing quality references). Grades focus on how well students critically assess their contributions in the context of the medium and the subject matter. As for the literature review, drafts of this task are peer reviewed before final submission.

\section{Self-Reflection Essay}

This task requires students to reflect on the objectives of the assignment, particularly whether and how material related to their research essay is presented on the platform and the implications of this. They are also expected to assess their own learning preferences, strengths, and styles by reflecting on their contributions (to both Wikipedia and their group), evaluating the skills they developed or improved throughout the assignment, and contemplating the challenges they faced or the activities with limited value. This task is not peer reviewed.

\section{Attendance}

Given the emphasis on group discussions and collaboration for this assignment, a nominal attendance grade is granted for participating in a certain percentage of the workshops.

\section{OUTCOMES}

This assignment surpassed my expectations in fulfilling the objectives outlined previously, as evidenced by student development observed during the semester and student feedback throughout the course. In student evaluations and self-reflections, an overwhelming majority of students reported that they had a more nuanced understanding of how Wikipedia entries are constructed, the epistemologies that it privileges, and a better 
sense of how they might thoughtfully engage with the medium. Many noted that they were now more skeptical consumers of Wikipedia content, with an improved ability to quickly assess whether they were willing to trust or use the information presented on a particular page. They were also more attentive to what information might be missing from an entry. Some mentioned that this scrutiny generalized beyond Wikipedia to other sources.

A striking feature of this assignment was that it substantially changed the topics that students chose for their research paper compared with previous years. Although papers still covered topics such as prime-ministerial leadership and major policy events, there were many more that investigated less-wellresearched topics including policy decisions affecting minorities, recognized obstacles to their contributions is heartening-on reflection, they might engage in future editing where they otherwise might not, or they simply may be more attuned to their positionality in society and the obstacles that this might present. However, the fact that only a few vocalized this concern during the semester suggests that more needs to be done to address the problem in class. It also raises concerns about the burden being placed on female and minority students, especially with respect to the additional gendered and emotional labor requested of them (Menking and Erickson 2015).

Another challenge is the extent to which it is possible to diversify Wikipedia beyond the sheer scope of the task. For example, one irreconcilable issue in using Wikipedia to present indigenous perspectives is that there are aspects of indigenous

\section{Rather than taking an abstinence approach to Wikipedia, interrogating the medium for strengths and weaknesses encourages students to be critical consumers of the content presented on its pages.}

indigenous politics, and less-well-known leaders and events. Research papers also tended to rely on more primary sources and original research than in previous years, suggesting that students were more attuned to how source quality affects research validity.

A major success was the excitement students expressed for engaging in an assignment with "real-world" applications and their satisfaction in producing original research that could be communicated to a wider audience. Former students have told me that they used this assignment in job interviews as evidence of skills such as writing for a public audience and critical analysis. Many were proud to share their Wikipedia edits with friends and family.

There has been little negative feedback on this assignment. Some students noted that it resulted in a heavier workload than courses with more traditional assessment. In all but one case, however, these students also acknowledged that the assignment was valuable and rewarding.

\section{CHALLENGES}

In their self-reflection essays, several female and minority students mentioned that they lacked confidence to make contributions as significant as they would have liked. Their contributions matched these observations-they were less likely than their male counterparts to start a new article or to contribute large sections of original text, especially if they were students in the lower-grade ranges. This occurred despite explicit instruction showing that marginalized voices were missing from the platform and encouragement for students to recognize and act on this.

Reasons for the gender gap on Wikipedia are well documented and include aspects of the editing process being particularly unappealing to females, such as high levels of conflict and criticism (especially with respect to gender-related topics; see Jemielniak 2016), as well as females reporting lower levels of confidence in their expertise (Collier and Bear 2016). Little is known about differences in Wikipedia contributors based on other characteristics. The fact that a number of students knowledge that are inherently incompatible with the platform. The mere fact of it being a written medium privileges written knowledge and "verifiable" (i.e., written) sources, and some indigenous knowledge simply should not be shared publicly (Timperley 2017). Although I did not encounter these specific issues, they are worthy of sustained attention and could productively inform discussion with students about the reliability and suitability of sources for particular types of knowledge, the effects on power relations, and the suitability of using Wikipedia as a resource for improving access to marginalized content. More work must be undertaken to examine whether Wikipedia is inherently antithetical to objectives such as decolonizationthat is, whether the platform design is an obstacle to such objectives-or whether the content that emerges signifies something more general about the culture of knowledge production in the world.

\section{MODIFICATIONS}

Although there was extensive discussion with students about the challenges in contributing content to Wikipedia-not least of which might be confidence of the editors themselves-more could be done to overcome the gender and minority confidence gap. Inviting a diverse panel of students from the previous year's course to speak with students about their experiences in completing the assignment might demonstrate its viability to those uncertain about their ability to contribute. Using anonymous surveys throughout the semester to determine whether students are struggling with particular parts of the assignment might help to overcome obstacles to contributing. Similarly, dedicating time to reflect in workshop groups about how to address controversial materials and challenges also might mitigate potentially damaging effects of "wiki lawyering" 4 on students new to editing.

Although I developed this assignment for a New Zealand politics course, the principles are clearly applicable in other courses, and it could be adapted to address topics that are underserved as a result of lack of interest or contributor expertise, not only those that arise from inequalities in power relations. 
In the United States, there have been calls for the decolonization and diversification of academic courses, including demands within political science to address the exclusion of American Indians (Ferguson 2016) and other minority voices (Colgan 2017; McClain et al. 2016). Courses across subfields (and levels) could benefit from considering whose voices and perspectives are omitted or underrepresented and then working to make Wikipedia more representative in its presentation of political science material.

Rather than taking an abstinence approach to Wikipedia, interrogating the medium for strengths and weaknesses encourages students to be critical consumers of the content presented on its pages. Recognizing Wikipedia as an imperfect source of information but with potential for revision is a critical way in which political scientists might work toward more accurate representation of political science material on one of the most widely used sources of information in the world.

\section{NOTES}

1. I use the language of decolonization with apprehension, acknowledging Tuck and Yang's seminal thesis (2012) that "decolonization is not a metaphor," while also recognizing that the language of decolonization has been used to make legible certain claims and actions to a broader audience.

2. Good resources include those available at https://dashboard.wikiedu.org/ training/students and www.artandfeminism.org.

3. An alternative would be to ask students to submit links to summaries of page edits, which would allow the instructor to assess their contributions in light of other edits to the page-especially if such edits proved controversial. They also could be required to include metrics such as page views (using, e.g., https://tools.wmflabs.org/pageviews/?project=en.wikipedia.org\&platform=all access\&agent=user\&range=latest-2o\&pages) to measure impact. This would be valuable for students who want to see how many readers are viewing their work-although it could affect which topics they choose to edit. Thank you to an anonymous reviewer for these suggestions.

4. This term is used to describe the way that seasoned editors challenge lessexperienced editors using their superior knowledge of Wikipedia's policies.

\section{REFERENCES}

Anthony, Denise, Sean W. Smith, and Timothy Williamson. 2009. "Reputation and Reliability in Collective Goods: The Case of the Online Encyclopedia Wikipedia." Rationality and Society 21 (3): 283-306.

Bayliss, Gemma. 2013. "Exploring the Cautionary Attitude Toward Wikipedia in High Education.” New Review of Academic Librarianship 19 (1): 36-57.

Brown, Adam R. 2011. "Wikipedia as a Data Source for Political Scientists: Accuracy and Completeness of Coverage." PS: Political Science $\mathcal{E}$ Politics 44 (2): $339-43$

Bruyneel, Kevin. 2014. "Social Science and the Study of Indigenous People's Politics: Contributions, Omissions, and Tensions." In Oxford Handbook of
Indigenous People's Politics, ed. José Antonio Lucero, Dale Turner, and Donna Lee VanCott. Oxford Handbooks Online.

Cassell, Mark K. 2018. "When the World Helps Teach Your Class: Using Wikipedia to Teach Controversial Issues." PS: Political Science E Politics $51(2): 427-33$.

Colgan, Jeff. 2017. "Gender Bias in International Relations Graduate Education? New Evidence from Syllabi.” PS: Political Science \& Politics 5o (2): 456-6o.

Collier, Benjamin, and Julia Bear. 2016. "Where Are the Women in Wikipedia? Understanding the Different Psychological Experiences of Men and Women in Wikipedia." Sex Roles 74 (5-6): 254-65

Di Lauro, Frances, and Rebecca Johinke. 2017. "Employing Wikipedia for Good Not Evil: Innovative Approaches to Collaborative Writing Assessment.” Assessment $\mathcal{E}$ Evaluation in Higher Education 42 (3): 478-91.

Fallis, Don. 2008. "Toward an Epistemology of Wikipedia." Journal of the American Society for Information Science and Technology 59 (10): 1662-74.

Ferguson, Kennan. 2016. "Why Does Political Science Hate American Indians?" Perspectives on Politics 14 (4): 1029-38.

Ford, Heather, and Judy Wajcman. 2017. “'Anyone Can Edit,' Not Everyone Does: Wikipedia's Infrastructure and the Gender Gap." Social Studies of Science 47 (4): 511-27.

Gallert, Peter, and Maja van der Velden. 2015. "Reliable Sources for Indigenous Knowledge: Dissecting Wikipedia's Catch-22.” In At the Intersection of Indigenous and Traditional Knowledge and Technology Design, ed. Nicola J. Bidwell and Heike Winschiers-Theophilus, 117-32. Santa Rosa, CA: Informing Science.

Gruwell, Leigh. 2015. "Wikipedia's Politics of Exclusion: Gender, Epistemology, and Feminist Rhetorical (In)action." Computers and Composition 37: 117-31.

Jemielniak, Dariusz. 2016. "Breaking the Glass Ceiling on Wikipedia." Feminist Review 113 (1): 103-108.

Jennings, Eric. 2008. "Using Wikipedia to Teach Information Literacy." College $\mathcal{E}$ Undergraduate Libraries 15 (4): 432-37

Kennedy, Ryan, Eric Forbush, Brian Keegan, and David Lazer. 2015. “Turning Introductory Comparative Politics and Elections Courses into Social Science Research Communities Using Wikipedia.” PS: Political Science \& Politics 48 (2): 378-83.

Knight, Charles, and Sam Pryke. 2012. "Wikipedia and the University, a Case Study." Teaching in Higher Education 17 (6): 649-59.

McClain, Paula D., Gloria Y. A. Ayee, Taneisha N. Means, Alicia M. Reyes-Barriéntez, and Nura A. Sediqe. 2016. "Race, Power and Knowledge: Tracing the Roots of Exclusion in the Development of Political Science in the United States." Politics, Groups, and Identities 4 (3): 467-82.

Menking, Amanda, and Ingrid Erickson. 2015. "The Heart Work of Wikipedia: Gendered, Emotional Labor in the World's Largest Online Encyclopedia." CHI 2015-Crossings: 207-10.

Smith, Linda Tuhiwai. 1999. Decolonizing Methodologies: Research and Indigenous Peoples. Dunedin, NZ: University of Otago Press.

Timperley, Claire. 2017. "Justice in Indigenous Land Claims: A Typology of Problems." Politics, Groups, and Identities. Available at DOI: 10.108o/21565503.2017.1411282.

Tuck, Eve, and K. Wayne Yang. 2012. "Decolonization Is Not a Metaphor.” Decolonization: Indigeneity, Education \& Society 1 (1): 1-40.

Wadhwa, Kul, and Howie Fung. 2014. "Converting Western Internet to Indigenous Internet: Lessons from Wikipedia." Innovations 9 (3/4): 127-35.

Wagner, Claudia, Eduardo Graells-Garrido, David Garcia, and Filippo Menczer. 2016. "Women through the Glass Ceiling: Gender Asymmetries in Wikipedia." EPJ Data Science 5:5. 